\section{Changes in ocular flow induced by hypo- and hypercapnia relate to static visual acuity in humans}

\author{
Naoyuki Hayashi, Tsukasa Ikemura, \\ Nami Someya \\ Institute of Health Science and Graduate \\ School of Human-Environment Studies, \\ Kyushu University, Kasuga, Japan
}

\section{Abstract}

We investigated whether the change in ocular blood flow, induced by hypo- and hypercapnia, is related to static visual acuity. Eleven healthy subjects ( $26 \pm 5$ years) underwent three treatments. A three-treatment three-period crossover design was used. In the hypocapnia treatment (HYPO), the subjects controlled their minute ventilation $\left(\mathrm{V}_{\mathrm{E}}\right)$ to a target of 25 $\mathrm{L} / \mathrm{min}$ for $6 \mathrm{~min}$. In the hypercapnia treatment (HYPER), the subjects inspired high-fraction $\mathrm{CO}_{2}$ gas $\left(\mathrm{FICO}_{2}=4 \%\right)$ for $6 \mathrm{~min}$. In the control treatment (CON), $\mathrm{V}_{\mathrm{E}}$ was not manipulated. We measured choroidal and retinal blood flow by laser speckle flowmetry as ocular blood flow, and static visual acuity using the Landolt $\mathrm{C}$ chart. End-tidal partial pressure of $\mathrm{CO} 2$ differed significantly among HYPO, HYPER and CON $(21 \pm 1,48 \pm 1$, and $42 \pm 1 \mathrm{mmHg}$, respectively). Retinal blood flow decreased significantly from the baseline in HYPO $(-22 \pm 5 \%)$, but increased significantly in HYPER $(+3 \pm 9 \%)$ compared to CON. Decimal visual acuity was significantly lower in HYPO than in the CON (0.21 \pm 0.1 vs. $0.24 \pm 0.1 \mathrm{P}<0.05)$. These results suggest that changes in ocular blood flow induced by changes in arterial $\mathrm{CO}_{2}$ partial pressure influences visual acuity.

\section{Introduction}

A continuous blood supply to the retina is essential for the maintenance of eye function because $\mathrm{O}_{2}$ is in high demand and is not stored in the retina and surrounding tissue ${ }^{1}$. Nutrition to the retina comes from the retinal and choroidal vessels. ${ }^{2}$ Loss of ocular blood flow regulation associated with pathological conditions, such as diabetes and glaucoma, is related to impaired visual function. ${ }^{1-4}$ In extreme cases, loss of myelinated axons in the optic nerve is observed by the pharmacological decrease in ocular blood flow in rabbits; ${ }^{5}$ however, the effect of acute changes in ocular blood flow on visual function has not been elu- cidated in healthy humans. On the other hand, increase in choroidal blood flow was observed with improved contrast sensitivity in healthy subjects after sidenafil administration. ${ }^{6}$ Thus we can simply hypothesize that acute change in ocular blood flow alters vision. The main purpose of the present study was to examine the hypothesis that increased ocular blood flow improves visual acuity, and decreased ocular blood flow decreases visual acuity. The present study was designed to observe change in visual acuity and ocular blood flow associated with change in arterial $\mathrm{CO}_{2}$ partial pressure $\left(\mathrm{PaCO}_{2}\right)$ induced by change in ventilation. For functional relevance, the decrease in ocular blood flow could be attributed to impairment of visual acuity after high intensity exercise, ${ }^{7}$ which induces hyperventilation and consequent decrease in ocular flow. Additionally, we compared the $\mathrm{CO}_{2}$ sensitivity in ocular and cerebral blood vessels since it is well known that ocular blood vessels are, like cerebral blood vessels, very sensitive to variations in $\mathrm{PaCO}_{2}$. ${ }^{2,8,9,10}$

\section{Materials and Methods}

\section{Subjects}

Eleven healthy non-smokers ( 5 males and 6 females, $26 \pm 5$ years, $171 \pm 14 \mathrm{~cm}, 64 \pm 14 \mathrm{~kg}$, mean \pm SD) participated in this study. All subjects were free of any known autonomic dysfunction, cardiovascular and ocular disease, and were not using medications. The Ethics Committee of the Institution of Health Science, Kyushu University, Japan, approved the experimental protocol, and all subjects provided written informed consent to participate prior to the commencement of the study. All protocols conformed to the Declaration of Helsinki. Before the experiments, each subject visited the laboratory for familiarization with the techniques and procedures of the protocol.

\section{Procedures}

All experiments were performed at a room temperature at $24^{\circ} \mathrm{C}$, illuminated at $90-100 \mathrm{~lx}$. The subjects arrived at the laboratory after having abstained from caffeinated beverages and exercise for $6 \mathrm{~h}$, and from a light meal for at least $2 \mathrm{~h}$.

For instruction and probe setting, the subject rested in a chair. Two minutes of baseline data were recorded while the subjects breathed room air. A three-treatment three-period crossover design was used. All subjects performed three 6 -min experimental procedures after the baseline period, consisting of eupnea (CON), hypocapnia (HYPO) and hypercapnia (HYPER). Washout period was $30 \mathrm{~min}$ between treatments. In the $\mathrm{CON}$ treatment, ventilation
Correspondence: Naoyuki Hayashi, Institute of Health Science, Kyushu University, Kasuga, Fukuoka 816-8580, Japan.

Tel. +81.92.583.7848 - Fax: +81.92.583-7848.

E-mail: naohayashi@ihs.kyushu-u.ac.jp

Key words: ventilation, $\mathrm{CO}_{2}$, vision, ocular circulation.

Acknowledgement: the authors are grateful for a grant from the Yamaha Motor Foundation for Sports to NH.

Contributions: NH, study design, data acquisition and interpretation, article drafting, revising and final approval; TI, data analysis and article revising; NS, data acquisition and article revising.

Conflict of interest: the authors report no conflicts of interest.

Received for publication: 18 May 2011. Accepted for publication: 6 September 2011.

This work is licensed under a Creative Commons Attribution NonCommercial 3.0 License (CC BYNC 3.0).

(C) Copyright N.i Hayashi et al., 2011

Licensee PAGEPress, Italy

Eye Reports 2011; 1:e8

doi:10.4081/eye.2011.e8

was not controlled. In the HYPO treatment, the subjects controlled their minute ventilation $\left(\mathrm{V}_{\mathrm{E}}\right)$ at $25 \mathrm{~L} / \mathrm{min}$ with $1 \mathrm{~L}$ tidal volume and 25 times/min respiratory rate with auditory feedback using a metronome and an experimenter. In the HYPER treatment, the subjects inspired high-fraction $\mathrm{CO}_{2}$ gas $\left(\mathrm{FICO}_{2}=4 \%\right)$ from a Douglas bag. In all treatments, subjects inspired through a Y-shaped valve from a Douglas bag filled with the gas or air. The expired gas was sent to the outside. The subjects wore a nose clip. The order of the treatments was randomized. For allocation of the participants, a computer-generated list of random numbers was used. Subjects who wore glasses or contact lens took these off during the experiment.

\section{Measurement}

Standard electrocardiogram was recorded continuously (ECG; MEG2100; Nihon-kohden, Tokyo, Japan). Beat-by-beat blood pressure was monitored with an automatic sphygmomanometer on the left middle finger (Finometer; Finapres Medical System, Amsterdam, the Netherlands). The mean blood velocity (MCAV) of the right middle cerebral artery (MCA) was obtained by transcranial Doppler ultrasonography (WAKI; Atys Medical, Soucieu-en-Jarrest, France). A 2 MHz Doppler probe was placed on the right temporal window 
and fixed with an adjustable headband. The MCAV waveform was obtained 47-51 mm from the skin surface. The signals from the ECG, sphygmomanometer and MCAV were sampled and recorded at $1 \mathrm{kHz}$ using an $\mathrm{A} / \mathrm{D}$ converter (PowerLab 8/30; ADInstruments, C0, USA). Minute-by-minute heart rate and mean arterial pressure (MAP) were calculated from ECG and blood pressure recordings. The subjects breathed through a mouthpiece connected to a hot wire flowmeter (RM-300; Minato Medical Sciences, Osaka, Japan) for the measurement of $\mathrm{V}_{\mathrm{E}}$ and end-tidal $\mathrm{CO}_{2}$ partial pressure $\left(\mathrm{P}_{\mathrm{ET}} \mathrm{CO}_{2}\right)$. The flowmeter was calibrated using a 2-L syringe. A small sample of respired gas (1 $\mathrm{mL} / \mathrm{s}$ ) was continuously withdrawn from the mask and analyzed for $\mathrm{CO}_{2}$ with a mass spectrometer (WSMR-1400; Westron Corp., Chiba, Japan). The mass spectrometer was calibrated with fresh air and precision gas. Static visual acuity of the right eye was estimated for the last two minutes of the resting baseline and each gas inspiration using the Landolt $\mathrm{C}$ chart $1 \mathrm{~m}$ from the subjects' eyes. Three different charts were used randomly not to allow subjects to memorize the chart. Visual acuity was expressed as decimal acuity (no unit), i.e., 20:20 and 20:200 correspond to 1.0 and 0.1 , respectively.

Laser speckle for four seconds was obtained 3 or 4 times for 0.5 -2 min for the baseline and 4-6 min for each treatment (LSFG; SoftCare-Ltd, Fukuoka, Japan). Subjects were asked to open their right eye for more than four seconds during the measurement. No drug administration such as mydriatic was used. LSFG is a non-invasive technique to measure real-time, two-dimensional relative blood flow velocity of ocular microcirculation using the laser speckle phenomenon, ${ }^{11}$ which is recognized as an interference phenomenon of coherent light sources, such as lasers. ${ }^{12}$ When tissue is illuminated under laser radiation, a speckle pattern appears, the structure of which changes rapidly according to blood flow velocity. ${ }^{13} \mathrm{~A}$ laser beam emitted from a laser diode (wavelength $830 \mathrm{~nm}$ ) is transmitted into part of the intraocular tissue. The observation field included the optic nerve head and the macular area. The blood flow map is obtained from the mean blur rate (30 frames per second), a quantitative index of relative blood flow velocity. From the data, two-dimensional perfusion maps are determined (Figure 1), quantifying blood flow velocity using commercially available software (LSFG Analyzer ver. 3.0.33.0 SoftCare-Ltd, Fukuoka, Japan). The mean velocity of blood flow was calculated from three or more entire cardiac cycles. Ocular blood flow velocity was obtained from retinal and choroidal capillary vessels (RCV). RCV was obtained from a given observation field of capillary vessels between the optic nerve head and the macula. This field was the same among subjects. So RCV represents blood flow velocity. This measurement does not separate retinal and choroidal flow. Ocular blood flow was obtained from inferior temporal retinal arteriole (ITRA), and superior temporal retinal arteriole (STRA). The cross section of the target arteriole was automatically selected by the software, and the blood flow was calculated from the integral of a cross sectional map of perfusion. The software can identify the arteriole, but not the capillaries. Thus, it provides relative blood flow of the arterioles according to the integral of a cross sectional map of perfusion.

\section{Data analysis}

$\mathrm{PaCO}_{2}$ was estimated from $\mathrm{V}_{\mathrm{E}}$ and $\mathrm{P}_{\mathrm{ET}} \mathrm{CO}_{2} \cdot{ }^{14}$ Ocular blood flow measurements were used for analysis only when clear laser speckle imaging was obtained in two or more consecutive heart beats during a 4-s recording period. These flow data were averaged in each condition and calculated as a relative value to the resting baseline value. Data are expressed as the mean \pm SE. The effect of treatments was examined by repeated-measures ANOVA. When a significant $F$ value was detected, this was analyzed further against the CON value using Dunnett's post-hoc test. Likewise, the relative response from the resting baseline in each vasculature to different ventilations was compared by repeated-measures ANOVA and Dunnett's test to compare with the MCAV value. Pearson correlation coefficient was calculated for the responses to $\mathrm{CO}_{2}$ among vasculatures, and between acuity changes and relative changes in blood flow in various vasculatures. Multiple regression analysis was performed to reveal effects of blood flow in RCV and MCA on visual acuity. The level of statistical significance was set at $\mathrm{P}<0.05$. These sta-

Table 1. MAP, HR, MCAV, $\mathrm{P}_{\mathrm{a}} \mathrm{CO}_{2}$ and $\mathrm{V}_{\mathrm{E}}$ during baseline and treatment.

\begin{tabular}{|c|c|c|c|}
\hline & & Baseline & Treatment \\
\hline $\begin{array}{l}\mathrm{MAP}(\mathrm{mmHg}) \\
\mathrm{CON}\end{array}$ & $\begin{array}{l}\text { HYPER } \\
\text { HYPO } \\
87.4 \pm 3.2\end{array}$ & $\begin{array}{l}87.6 \pm 3.6 \\
87.4 \pm 3.8 \\
85.8 \pm 2.6\end{array}$ & $\begin{array}{l}88.9 \pm 3.7^{*} \\
84.5 \pm 3.6^{*}\end{array}$ \\
\hline HR (bpm) & $\begin{array}{l}\text { HYPER } \\
\text { HYPO } \\
\text { CON }\end{array}$ & $\begin{array}{l}65.7 \pm 3.7 \\
67.1 \pm 4.6 \\
63.9 \pm 3.5\end{array}$ & $\begin{array}{l}68.0 \pm 3.8^{*} \\
78.9 \pm 5.5^{*} \\
65.6 \pm 3.6\end{array}$ \\
\hline $\begin{array}{l}\mathrm{MCAV}(\mathrm{cm} / \mathrm{s}) \\
\mathrm{CON}\end{array}$ & $\begin{array}{l}\text { HYPER } \\
\text { HYPO } \\
79.3 \pm 4.3\end{array}$ & $\begin{array}{l}76.2 \pm 4.4 \\
79.1 \pm 3.8 \\
77.1 \pm 4.0\end{array}$ & $\begin{array}{l}81.7 \pm 4.9^{*} \\
46.1 \pm 2.6^{*}\end{array}$ \\
\hline $\mathrm{P}_{\mathrm{a}} \mathrm{CO}_{2}(\mathrm{mmHg})$ & $\begin{array}{l}\text { HYPER } \\
\text { HYPO } \\
\text { CON }\end{array}$ & $\begin{array}{l}42.5 \pm 1.3 \\
43.2 \pm 0.6 \\
43.1 \pm 1.4\end{array}$ & $\begin{array}{l}48.4 \pm 0.6^{*} \\
23.9 \pm 0.3^{*} \\
43.2 \pm 1.0\end{array}$ \\
\hline $\mathrm{V}_{\mathrm{E}}(\mathrm{L} / \mathrm{min})$ & HYPER & $6.8 \pm 0.4$ & $12.1 \pm 0.9 *$ \\
\hline $\mathrm{CON}$ & $\begin{array}{l}\text { HYPO } \\
7.0 \pm 0.4\end{array}$ & $\begin{array}{l}7.1 \pm 0.5 \\
7.7 \pm 0.4\end{array}$ & $22.9 \pm 1.4^{*}$ \\
\hline
\end{tabular}

Values are the mean \pm SE. All variables during the treatment significantly changed from the resting baseline values, except in the CON treatment. ${ }^{*} \mathrm{P}<0.05$ vs. baseline. tistical analyses were performed with SAS (version 8.2; SAS Institute, Cary, NC, USA) at the Computing and Communications Center, Kyushu University, Japan.

\section{Results}

\section{Systemic change}

Hypercapnia increased both MAP and HR, while hypocapnia decreased MAP and increased HR (Table 1). These changes were slight but significant. Hypercapnia significantly increased MCAV, while hypocapnia significantly decreased it (Table 1 ). $\mathrm{P}_{\mathrm{ET}} \mathrm{CO}_{2}$ differed

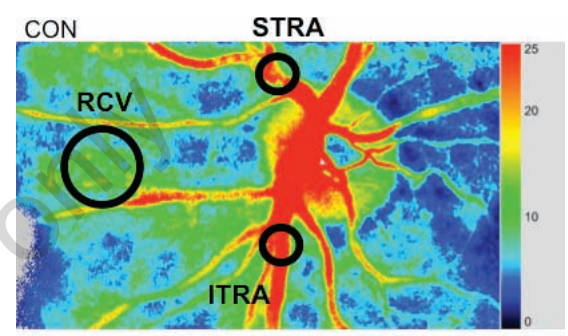

HYPO

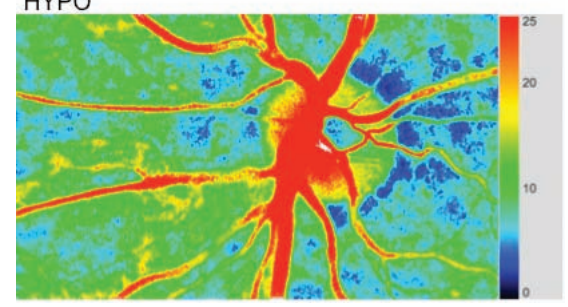

Figure 1. Examples of the velocity data obtained in a subject at eupnia (CON, upper) and hypocapnia (HYPO, bottom). Color bars shown in the right side indicates the blood flow velocity. Note decrease in blood flow velocity in HYPO, compared to $\mathrm{CON}$ treatment. 
significantly among CON, HYPO, and HYPER treatments $(42 \pm 1,21 \pm 1$, and $48 \pm 1 \mathrm{mmHg}$, respectively). Accordingly, there were significant differences in estimated $\mathrm{PaCO}_{2}$ among treatments (Table 1).

\section{Ocular and cerebral blood flow}

The eupnea (CON) did not significantly alter blood flow velocity in MCA, and blood flow in RCV, ITRA and STRA from the baseline value $(\mathrm{P}>0.1)$.

Hyperventilation resulting in hypocapnia (HYPO) significantly decreased blood flow velocity in MCA, blood flow velocity in RCV, and blood flow in ITRA and STRA by $42 \pm 4 \%$, $22 \pm 15 \%, 15 \pm 8 \%$ and $13 \pm 18 \%$, respectively (Figure 2). The decrease in MCAV was significantly greater than in other vasculatures. High-fraction $\mathrm{CO}_{2}$ gas inspiration (HYPER) slightly but significantly changed blood flow

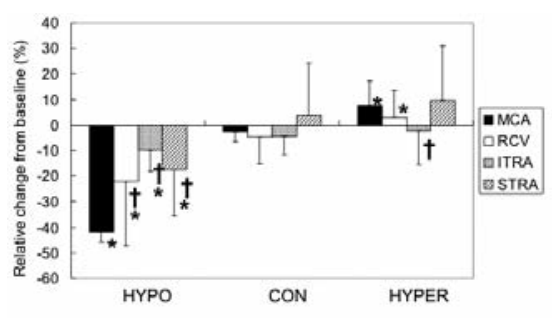

Figure 2. Relative changes in blood flow velocity in middle cerebral artery (MCAV), blood flow velocity in retinal and choroidal vessels (RCV), and blood flow in inferior temporal retinal arteriole (ITRA) and superior temporal retinal arteriole (STRA) in response to hypocapnia (HYPO), eupnia (CON) and hypercapnia (HYPER). HYPO significantly decreased blood flow in MCA, RCV, ITRA and STRA. The decrease in MCAV was significantly greater than in the other vasculatures. HYPER treatment slightly but significantly changed blood flow in MCA and RCV, but not in ITRA and STRA. The increase in MCAV was significantly greater than in ITRA. $n=11$. ${ }^{*} \mathrm{P}<0.05$ vs $\mathrm{CON},+\mathrm{P}<0.05$ vs $\mathrm{MCA}$

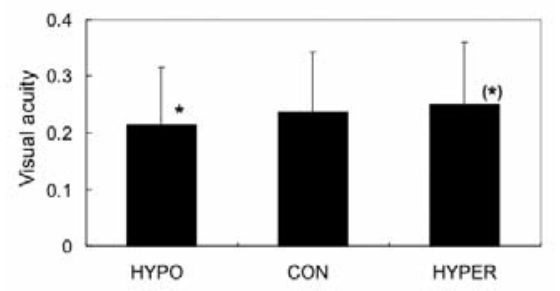

Figure 3. Visual acuity during hypocapnia (HYPO), eupnia (CON) and hypercapnia (HYPER). This was significantly less in HYPO and tended to be greater in HYPER than in the CON treatment. $\mathrm{n}=11 .{ }^{*} \mathrm{P}<0.05$ vs CON $\left({ }^{*}\right) \mathrm{P}=0.08$ velocity in MCA and RCV $(8 \pm 9 \%$ and $3 \pm 9 \%$, respectively), but not blood flow in ITRA and STRA. The increase in MCAV was significantly greater than in ITRA flow.

\section{Visual acuity}

Visual acuity with HYPO, CON and HYPER treatments was $0.21 \pm 0.1,0.24 \pm 0.1$ and $0.25 \pm 0.1$, respectively (Figure 3 ). Visual acuity was significantly lower in HYPO and tended to be greater in HYPER than in the CON treatment. The difference in visual acuity in HYPO and HYPER treatments compared with the CON treatment was significantly and positively correlated with the relative changes in blood flow in RCV and MCA ( $\mathrm{r}=0.52$ and 0.51 , respectively, $n=22$ ) (Figure 4). The correlation was still observed between visual acuity and RCV in HYPO treatment $(r=0.67, n=11)$. Except for this case, there was no significant correlation in changes between blood flow and visual acuity when HYPO and HYPER were separately examined. However, when deleting an extreme point in RCV and visual acuity relationship, we lost the significant correlation. On the other hand, in the MCA and visual acuity relationship, while even after deleting one to five extreme points, significant correlation was still present between MCA and visual acuity relationship. Multiple regression revealed the significant effect on a change in visual acuity of changes in blood flow both in MCA and RCV: $1,000 \mathrm{~V}=0.7 \mathrm{R}+0.4 \mathrm{M}+2.3$; where $\mathrm{V}$ represents the change in visual acuity from baseline, $\mathrm{R}$ and $\mathrm{M}$ are the relative changes in $\mathrm{RCV}$ blood flow and MCAV, respectively.

\section{Relationship in $\mathrm{CO}_{2}$ reactivity among vasculatures}

The correlation coefficient among relative responses in vasculature to HYPO treatment
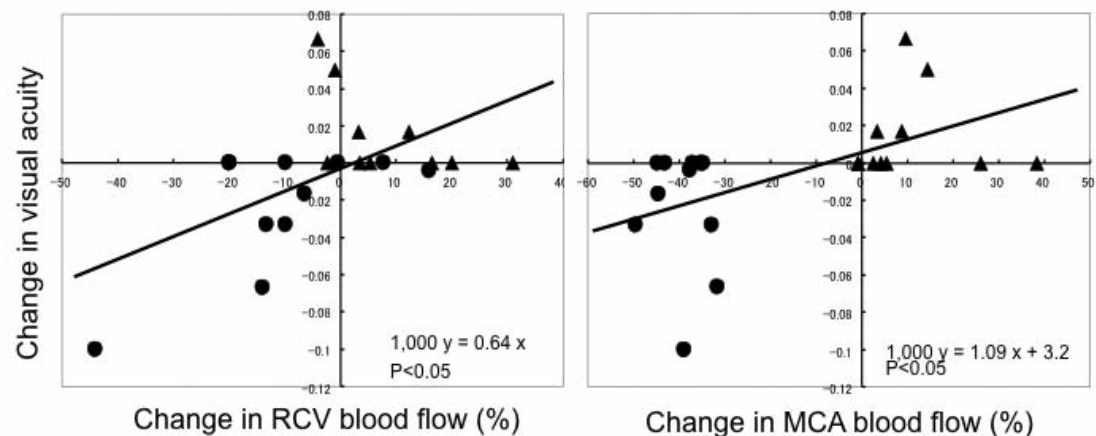

Change in MCA blood flow (\%)

was significant between ITRA and RCV $(\mathrm{r}=0.60)$, but no significant correlations were obtained among other ocular and cerebral blood flow responses ( $\mathrm{r}$ ranged from -0.04 to $0.50, \mathrm{P}>0.1$ ).

\section{Discussion}

A main finding in the present study is that hypo- and hypercapnia altered RCV with a concomitant change in static visual acuity, supporting our hypothesis that RCV correlates with static visual acuity. Another finding was that the magnitude of vessels response to $\mathrm{CO}_{2}$ in ocular vessels is less than in cerebral one, supporting a previous study which reported MCA blood flow response was greater to hypercapnia than retinal blood flow. ${ }^{15}$

Effect of ocular circulation on visual acuity The present results demonstrate for the first time that both acute increase and decrease of RCV correlate with the improvement and impairment of visual acuity in healthy humans, supporting our hypothesis. There was also a significant positive correlation between RCV and visual acuity. The finding is consistent with previous findings that increased blood flow was related to the improvement of contrast sensitivity. ${ }^{16}$ Concomitant increases in choroid flow and contrast sensitivity were found in healthy subjects after sildenafil administration. ${ }^{6}$ These findings could be supported by evidence for the relationship between chronic change in ocular blood flow and vision, ${ }^{1-4}$ though some previous studies have reported no relationship between ocular blood flow and visual function during hypoand hypercapnic, pharmacologic and altitude stimulus. ${ }^{17,18}$ Chronic reduction in ocular blood flow is well known to be related to impaired

Figure 4. Relationship between changes in visual acuity and blood flow velocity in the retinal and choroidal vessels (RCV, left panel) and middle cerebral artery (MCA, right). The difference in visual acuity from the eupnea (CON) treatment was significantly correlated with the relative changes of blood flow velocity in RCV and MCA $(r=0.52$ and 0.51 , respectively, $n=22, P<0.05)$. Circles show the response to hypocapnea $(\mathrm{HYPO})$ and triangles show that to hypercapnea (HYPER). 
visual function in diseases and animal models. ${ }^{1-5}$ Acute loss of contrast sensitivity in glaucoma patients was improved by hypercapnia. ${ }^{19}$ These findings could support the relevance of acute change in ocular blood flow on the concomitant change in visual acuity.

The relationship between changes in ocular flow and visual acuity could provide an important translational implication, if ocular blood flow is relevant to various visual variables, such as dynamic visual acuity, visual field and contrast sensitivity as well as static visual acuity. For example, the present finding is useful to find how to maintain or improve visual acuity during work. It was reported that high intensity exercise induces temporary impairment of visual acuity. ${ }^{7}$ High intensity exercise relates to hyperventilation, which induces hypocapnea. Thus the relationship of ocular blood flow to visual acuity has implications in preventing the decreased vision.

\section{Effect of $\mathrm{CO}_{2}$ on ocular and cere- bral vascular responses}

Blood flow velocity reduced in both ocular capillary and arterioles in response to decreased $\mathrm{PaCO}_{2}$, and partly increased in response to increased $\mathrm{PaCO}_{2}$. The response of ocular blood flow or blood flow velocity to hypercapnia has been well documented in retinal capillary, superior temporal peripapillary retina, and ophthalmic and central retinal arteries. . $^{18,20,21}$ The response to hypocapnia was also reported. ${ }^{18}$ The present study supports that both retinal arteriole and retinal-choroidal vasculatures respond to both hypo- and hypercapnia.

The response to hypo- and hypercapnia was greater in MCAV than blood flow velocity in ocular vessels and blood flow in retinal arterioles both in groups and individuals, indicating weaker reactivity in ocular than the cerebral vessels. Few previous studies have compared the ocular vessel and cerebral vessel response to $\mathrm{CO}_{2} \cdot{ }^{15,22,23}$ A weaker reactivity in ocular vessels to $\mathrm{CO}_{2}$ has physiological relevance. The function of the retina is vulnerable to overperfusion. ${ }^{2,9}$ Weak reactivity could serve for the relatively easy stabilization of ocular perfusion.

\section{Relationship between responses in various vessels}

Retinal vessels are routinely inspected during physical examinations and used to estimate clinical cerebral vessel conditions. A correlation between vascular reactivity to $\mathrm{CO}_{2}$ in retinal and cerebral vessels has been reported. ${ }^{24}$ Studies have been performed based on analogy of ocular vessels to the cerebral circulation; however, no published study to our knowledge has investigated the relationship between cerebral and ocular vessel responses to $\mathrm{CO}_{2}$. The present result does not show a relationship among these responses. This implies that the magnitude of response to vasoconstrictive and vasodilative mechanisms is not necessarily the same in ocular and cerebral vasculatures in an individual.

Limited correlation even in ocular flow responses might be due to the effect of this complex flow nature in the retina. RCV is a combination of retinal and choroidal flow velocity; the retinal circulation component is characterized by a low level of flow and is supplied by the central retinal artery, which is connected to the ITRA and the STRA, whereas the choroidal circulation component has relatively higher flow and is supplied by ciliary arteries. $^{9,25}$ Unfortunately, we cannot separate these signals in the present study.

\section{Limitations}

The increase in $\mathrm{PaCO}_{2}$ was limited since increase in inspired $\mathrm{CO}_{2}$ stimulates ventilation. HYPER treatment did not provide a corresponding change in $\mathrm{PaCO}_{2}$ to HYPO treatment. As a result, the decrease of ocular blood flow was limited and the change of visual acuity did not reach statistical significance. Increase in ventilation could increase $\mathrm{PaO}_{2}$. In our preliminary measurement in the same experimental setting, we observed $10 \%$ increase in $\mathrm{P}_{\mathrm{ET}} \mathrm{O}_{2}$ in four of subjects during HYPO treatment. An increase of $13 \%$ in $\mathrm{P}_{\mathrm{ET}} \mathrm{O}_{2}$ has been reported during hypercapnia. ${ }^{26}$ Thus, arterial oxygen tension accompanied by increased ventilation might reduce the ocular blood flow. Results obtained during hypocapnia could be mixed with the effect of increased oxygen tension. However the effect of oxygen tension is more limited than previous studies comparing ocular blood flow between air and carbogen. Using carbogen leads $\mathrm{P}_{\mathrm{ET}} \mathrm{O}_{2}$ up to $400 \mathrm{mmHg}$, ${ }^{27}$ while the present study might have roughly increase it by $115 \mathrm{mmHg}$. In turn, we can conclude that hypercapnea increases ocular blood flow since the effect of hypercapnia overwhelms the effect of increased oxygen, if any.

It should be noted that the change of $\mathrm{PCO}_{2}$ also alters the oxygen dissociation curve of hemoglobin (Hb), i.e., the Bohr effect and the converse of the Bohr effect. In fact, the possibility of impeding $\mathrm{O}_{2}$ unloading even in human muscle tissues has been reported. ${ }^{28}$ The present study does not allow us to conclude that the single effect of blood flow alters the visual acuity. In turn, there remains the possibility that the shift of oxygen dissociation curve of $\mathrm{Hb}$ modified the acuity; any factor modifying visual acuity associated with respiratory manipulation should be carefully interpreted.

Altered cerebral perfusion could affect visual acuity as shown in the concomitant change and significant correlation in changes of MCAV and visual acuity, though MCA dynamics may be different in nature than posterior cerebral artery dynamics. ${ }^{29}$ There is also indirect evidence that altered cerebral perfusion could affect visual acuity: a relationship between MCA blood flow and visual acuity and contrast sensitivity in primary open-angle glaucoma in a cross-sectional study. ${ }^{4}$ It is difficult to obtain direct evidence that cerebral perfusion relates to visual function because the nature of both cerebral and ocular flows are similar to many physiological stimulations. Thus there is room for argument as to whether or not cerebral blood flow changes the visual acuity.

In summary, we compared the ocular and cerebral blood flow among hyper-, hypo-, and normo-capnia conditions. We demonstrated that the ocular blood flow change induced by both hypo- and hyper-capnia are associated with concomitant changes in visual acuity. These findings are useful for further understanding the physiological nature of ocular blood flow and its relation to visual function.

\section{References}

1. Wangsa-Wiraman ND, Linsenmeier RA. Retinal oxygen. Fundamental and clinical aspects. Arch 0phthalmol 2003;121:547-57.

2. Bill A. Blood circulation and fluid dynamics in the eye. Physiol Rev 1975;55:383-417.

3. Guan K, Hudson C, Wong T, et al. Retinal hemodynamics in early diabetic macular edema. Diabetes 2006;55:813-8.

4. Harris A, Siesky B, Zarfati D, et al. Relationship of cerebral blood flow and central visual function in primary openangle glaucoma. J Glaucoma 2007;16:15963.

5. Sasaoka M, Taniguchi T, Shimazawa M, et al. Intravitreal injection of endothelin-1 caused optic nerve damage following to ocular hypoperfusion in rabbits. Exp Eye Res 2006;83:629-37.

6. Sponsel WE, Paris G, Sandoval SS, et al. Sildenafil and ocular perfusion. N Engl J Med 2000;342:1680.

7. Ishigaki H, Miyao M, Ishihara S, et al. The deterioration of visual acuity by exercise under a mesopic vision environment. J Sports Med Phys Fitness 1991;31:272-6.

8. Alm A, Bill A. The oxygen supply to the retina. I. Effects of changes in intraocular and arterial blood pressures, and in arterial P02 and PCO2 on the oxygen tension in the vitreous body of the cat. Acta Physiol Scand 1972;84:261-74.

9. Delaey C, Van de Voorde J. Regulatory mechanisms in the retinal and choroidal circulation. Opthalmic Res 2000;32:249-56.

10. Van De Borne P, Mezzetti S, Montano N, et al. Hyperventilation alters arterial baroreflex control of heart rate and muscle sym- 
pathetic nerve activity. Am J Physiol Heart Circ Physiol 2000;279:H536-41.

11. Tamaki Y, Araie M, Kawamoto E, et al. Non-contact, two-dimensional measurement of retinal microcirculation using laser speckle phenomenon. Invest Ophthalmol Vis Sci 1994;35:3825-34.

12. Tamaki Y, Araie M, Kawamoto E, et al. Non-contact, two-dimensional measurement of tissue circulation in the choroids and optic nerve head using laser speckle phenomenon. Exp Eye Res 1995;60:373-83.

13. Fujii H, Nohira K, Yamamoto $Y$, et al. Evaluation of blood flow by laser speckle image sensing. Part 1. Appl Opt 1987;26:5321-5.

14. Jones NL, Robertson DG, Kane JW. Difference between end-tidal and arterial PC02 in exercise. J Appl Physiol 1979;47:954-60.

15. Kisilevsky M, Mardimae A, Slessarev M, et al. Retinal arteriolar and middle cerebral artery responses to combined hypercarbic/hyperoxic stimuli. Invest Ophthalmol Vis Sci 2008;49:5503-9.

16. Huber KK, Adams H, Remky A, Arend KO. Retrobulbar haemodynamics and contrast sensitivity improvements after $\mathrm{CO} 2$ breathing. Acta Ophthalmol Scand 2006;84:481-7.

17. Bosch MM, Merz TM, Barthelmes D, et al. New insights into ocular blood flow at very high altitudes. J Appl Physiol 2009;106: 454-60.

18. Harris A, Arend 0 , Wolf S, et al. CO2 dependence of retinal arterial and capillary blood velocity. Acta Ophthalmol Scand 1995;73:421-4.

19. Hosking SL, Evans DW, Embleton SJ, et al. Hypercapnia invokes an acute loss of contrast sensitivity in untreated glaucoma patients. Br J Ophthalmol 2001;85:1352-6.

20. Chung HS, Harris A, Halter PJ, et al. Regional differences in retinal vascular reactivity. Invest Ophthalmol Vis Sci 1999;40:2448-53.

21. Lietz A, Hendrickson P, Flammer J, et al. Effect of carbogen, oxygen and intraocular pressure on Heidelberg retina flowmeter parameter 'flow' measured at the papilla. Ophthalmologica 1998;212:149-52.

22. Schmetterer L, Findl 0, Strenn K, et al. Role of NO in the 02 and $\mathrm{CO} 2$ responsiveness of cerebral and ocular circulation in humans. Am J Physiol 1997;273:R2005-12. 23. Bayerle-Eder M, Wolzt M, Polska E, et al.
Hypercapnia-induced cerebral and ocular vasodilation is not altered by glibenclamide in humans. Am J Physiol Regul Integr Comp Physiol 2000;278:R1667-73.

24. Hickam JB, Schieve JF, Wilson WP. The relation between retinal and cerebral vascular reactivity in normal and arteriosclerotic subjects. Circulation 1953;7:84-7.

25. Netter FH. Atlas of human anatomy 4 th ed. Philadelphia, PA: Saunders; 2006.

26. Venkataraman ST, Hudson C, Fisher JA, Flanagan JG. The impact of hypercapnia on retinal capillary blood flow assessed by scanning laser Doppler flowmetry. Microvasc Res 2005;69:149-55.

27. Luksch A, Garhöfer G, Imhof A, et al. Effect of inhalation of different mixtures of 02 and $\mathrm{CO} 2$ on retinal blood flow. Br J Ophthalmol 2002;86:1143-7.

28. Hayashi N, Ishihara M, Tanaka A, Yoshida T. Impeding 02 unloading in muscle delays oxygen uptake response to exercise onset in humans. Am J Physiol 1999;277:R127481.

29. Haubrich C, Wendt A, Diehl RR, Klötzsch C. Dynamic autoregulation testing in the posterior cerebral artery. Stroke 2004;35: 848-52. 